INTERNATIONAL JOURNAL OF RESEARCHES IN BIOSCIENCES, AGRICULTURE AND TECHNOLOGY (C) VISHWASHANTI MULTIPURPOSE SOCIETY (Global Peace Multipurpose Society) R. No.MH-659/13(N) www.vmsindia.org

\title{
ETHNOMEDICINAL SURVEY OF ARMORI, WADSA, KURKHEDA, KORCHI FOREST RANGE OF GADCHIROLI DISTRICT, MAHARASHTRA STATE, INDIA.
}

\section{S. Khonde ${ }^{1}$, M.C. Kale ${ }^{2}$ and R.S. Badere ${ }^{3}$}

${ }_{1}$ Department of Botany Raje Dharmarao College of Science Aheri. Anand Niketan College Anandwan, Warora. 3PGTD of Botany, Campus Nagpur. vijay.khonde160@gmail.com

\section{Abstract:}

This paper aims to investigate medicinal plant, used traditionally by the Gond and Madiya community. Reports from plant informants were obtained during field studies in January 2013 to January 2016. The botanical names, local name(s), medicinal uses, as well as plant part (s) used, and other uses with method of preparation. 100 plant species was recorded during this survey. The popularity of plant with traditional uses among the tribal people is fading due to migration, restriction from religion and dependence on modern medicine for the therapy. Use of forest land for agricultural development and timber harvesting is also contributing to the loss of knowledge. Awareness activities in Gadchiroli district among tribal people on the usefulness of medicinal plants has contributed significantly towards the preservation of old traditional knowledge of medicinal plants.

Keywords: Medicinal plants, Kurkheda, Korchi. Ethnobotany, Gond ,Madiya.

\section{Introduction:}

Man has been using plants from ancient time and research work constantly brings to light additional information on the relationship between plants and man. The theme of ethnobotany or folkloric botany reveals interrelation of plants and man. Plants have played a key role in day-to-day life support system of human being from time immemorial. Plants are an important source of traditional medicine for the treatment of various diseases. It has been estimated that herbal medicines are used by more than $80 \%$ of the world population in developing countries to meet their primary health care needs. In Gadchiroli district, especially in backward areas, the available modern healthcare services are either insufficient or inaccessible and unaffordable to the majority of people.In addition, due to illiteracy and economic status most of the population is dependent on traditional phytomedicine to cure various ailments. As the country has diverse socioeconomic, ethnic and cultural areas as well as unique biodiversity, knowledge of indigenous medicinal plant and their use in treating human ailments might reasonably be expected.

Gadchiroli district is situated on the NorthEastern side of the Maharashtra State in country India \& is well known for dense forest: having State borders of Andhra Pradesh and Chhattisgarh. The district is covered with hills and forests and is considered as a tribal area. The District falls under assured and heavy rainfall zone. The rain, are mainly received from South-West mansoon. The average rainfall is $1562 \mathrm{~mm}$. The climatic conditions are extreme with temperature reaching $47.30 \mathrm{C}$ in Summer \&
9.4oC in Winter. The District is blessed with huge forest and mineral resources. The forests are Predominantly in Etapalli, Aheri, Dhanora, Korchi, Kurkheda, Sironcha and Bhamragad blocks. The forest, are rich in Teak (Tectonagrandis), Ain (Terminalia tomentosa), Tendoo (Diospyrosmelan oxylon), Dhavada (Anogeissusl atifolia), Anjan (Memecylonum bellatum), etc. Ain and Anjan Similarly various plants having great medicinal values is available in large quantity and the climatic condition supports the development of these plants. The present work has been undertaken to identify the medicinal plants and their part used as medicine and the method of drug preparation.

\section{Study Area:}

The present work was carried with the tribal community of Gond, Madiaya, Pardhan, Halba, Halbi, Gowari. Located in villages of Kurkheda Jungaon patch, Bhagawanpur, Kudakwahi, Petedongari, Sawargaon.Korchi, Phakanbhatti, Nawegaon, Gutekasa, Kochinara, Surwahi, Mohagaon. InKurkheda and Korchi forest region. The gadchiroli district which covers the total area of about $14412 \mathrm{Km}^{2}$.

\section{Methodology:}

Regular field surveys were carried out in the Kurkheda and Korchi forest range form January 2013 through January 2016 in order to document the habitats and indigenous uses of ethnomedicinal plants of the forest valley. The surveys were carried out at different seasons so as to obtain identifiable plants and multiple information and also to cross-check the information provided by the local information provided by the local informants during earlier visits. We interviewed a small group of chiefly 
elder people of both Gond and Madiya tribes who were highly esteemed in their societies, due to their sound knowledge of medicinal plants. Structured questionnaires, formal and informal interviews and participatory observations were used plant part and the process of remedy preparation. This kind of information about medicinal plants indicates how a given medicine can be therapeutically efficient in term of the right ingredients, the proper dose and right duration of medication. According to tribal people their knowledge of folk medicine was acquired mainly through parents and experience about medicinal value of plant. Species were identified using relevant and standard literature.

Table. 1. Studies on Ethnomedicinal plants from Armori, Wadsa, Kurkheda, Korchi Forest Area.

\begin{tabular}{|c|c|c|c|c|c|c|}
\hline $\begin{array}{l}\text { Sr. } \\
\text { No }\end{array}$ & Botanical Name & Family & $\begin{array}{l}\text { Common } \\
\text { Name }\end{array}$ & $\begin{array}{c}\text { Gond / Madiya } \\
\text { Name }\end{array}$ & plant part used & $\begin{array}{l}\text { Uses Against } \\
\text { Disease/ for } \\
\text { Disease }\end{array}$ \\
\hline \multirow{2}{*}{1} & \multirow{2}{*}{$\begin{array}{l}\text { Asteracan tha longifolia } \\
\text { (L.) Nees. }\end{array}$} & \multirow{2}{*}{ Acanthaceae } & \multirow{2}{*}{$\begin{array}{l}\text { Talimkhan } \\
\text { (katekolasa) }\end{array}$} & \multirow{2}{*}{ Talimkhanmarrha } & Root, & Urinary problems, \\
\hline & & & & & Leaves,Seeds. & Jaundice. \\
\hline 2 & Barleria prionitisL. ssp & Acanthaceae & Katekorante & Sonerimarrha & Leaves, Flowers & paralysis \\
\hline 3 & $\begin{array}{l}\text { Agave Americana L. } \\
\text { var. americana }\end{array}$ & Agavaceae & Ghaypat & Ghaypatmarrha & Leves, & Cough \\
\hline \multirow{3}{*}{4} & \multirow{3}{*}{$\begin{array}{l}\text { Achyranthus aspera L. } \\
\text { var. aspera }\end{array}$} & \multirow{3}{*}{ Amaranthaceae } & \multirow{3}{*}{ Kuthri } & \multirow{3}{*}{ Kuthrimarrha } & Root, & Mouth Ulcer, \\
\hline & & & & & $\begin{array}{l}\text { Bark, } \\
\text { Leaves. }\end{array}$ & $\begin{array}{l}\text { Eye disordercough } \\
\text { and in digestives }\end{array}$ \\
\hline & & & & & Whole plant & snakebite, piles \\
\hline 5 & $\begin{array}{c}\text { Amaranthus spinosus } \\
\text { L. }\end{array}$ & Amaranthaceae & $\begin{array}{l}\text { Kathematha or } \\
\text { ChavlichiBhaji }\end{array}$ & Doggelkusari & Leaves & Constipation \\
\hline \multirow{2}{*}{6} & \multirow{2}{*}{$\begin{array}{l}\text { Buchanania lanzan } \\
\text { Spreng. }\end{array}$} & \multirow{2}{*}{ Anacardiaceae } & \multirow{2}{*}{ Char } & \multirow{2}{*}{ Herkamarrha } & Root and & Arthritis, \\
\hline & & & & & Leaves & wound healing \\
\hline \multirow{3}{*}{7} & \multirow{3}{*}{ Annonas quamosa L. } & \multirow{3}{*}{ Annonaceae } & \multirow{3}{*}{ Shitaphal } & \multirow{3}{*}{ Shitaphalmarrha } & Fruits, & Bone marrow, \\
\hline & & & & & Roots, Leaves, & Healing of wound, \\
\hline & & & & & Seed. & Worms. \\
\hline 8 & $\begin{array}{l}\text { Rauvolfia serpentine (L) } \\
\text { Benth. Ex. Kurz. }\end{array}$ & Aopcynaceae & Sarpagandha & $\begin{array}{c}\text { Sarpagandhamarrh } \\
\text { a }\end{array}$ & $\begin{array}{l}\text { Dried Root } \\
\text { powder }\end{array}$ & $\begin{array}{l}\text { Snake Bite, } \\
\text { hypertension, } \\
\text { Insomnia and } \\
\text { insanity. }\end{array}$ \\
\hline 9 & $\begin{array}{c}\text { Centella asiatica (L.) } \\
\text { Urb. } \\
\end{array}$ & Apiaceae & Brahmi & Brahmimarrha & Plant & $\begin{array}{l}\text { Jaundice, Tonic, } \\
\text { Skin disease }\end{array}$ \\
\hline 10 & Coriandrum sativum $L$. & Apiaceae & Dhane & Dhanemarrha & Seed, Fruit & $\begin{array}{l}\text { Carminative } \\
\text { Rheumatic pain }\end{array}$ \\
\hline 11 & $\begin{array}{c}\text { Carissa congesta Wight } \\
\text { var. congesta }\end{array}$ & Apocynaceae & Karvand & Karvandmarrha & Root, Fruit & Malaria, Digestive \\
\hline \multirow{3}{*}{12} & \multirow{3}{*}{$\begin{array}{l}\text { Catharanthusroseus } \\
\text { (L).G.Don. }\end{array}$} & \multirow{3}{*}{ Apocynaceae } & \multirow{3}{*}{ Sadafuli } & \multirow{3}{*}{ Pungarmarrha } & Flowers, & Leukemia, \\
\hline & & & & & $\begin{array}{l}\text { Leaves, whole } \\
\text { Plant. }\end{array}$ & $\begin{array}{l}\text { plant used as } \\
\text { remedy for } \\
\text { diabetes. } \\
\text { Menstruation } \\
\text { cycle. }\end{array}$ \\
\hline & & & & & Roots, Flower. & Cancer. Diabetes. \\
\hline 13 & $\begin{array}{c}\text { Holarrhena } \\
\text { antidysentericasensu. } \\
\text { Wall. Ex. DC. }\end{array}$ & Apocynaceae & Safedkuda & Safedkuda, Marrha & Bark & Dysentery \\
\hline 14 & $\begin{array}{c}\text { Calatropis procera Ait } \\
\text { R. Br }\end{array}$ & Apocynaceae & Rui & Ruimarrha & Milk & Cough and Cold \\
\hline 15 & $\begin{array}{l}\text { Phoenix sylvestris (L.) } \\
\text { Roxb. }\end{array}$ & Arecaceae & Sindhi & Sindhimarrha & Fruit,Bark, & $\begin{array}{l}\text { Pails, } \\
\text { Arthritis, } \\
\text { Headache, Fever, } \\
\text { Tonic, Cold flu, } \\
\text { Pain killer }\end{array}$ \\
\hline 16 & $\begin{array}{c}\text { Colocasia esculenta (L.) } \\
\text { Schott }\end{array}$ & Arecaceae & Dhopa & Dhopamarrha & Leaves, Rhizome & Sex power \\
\hline \multirow[b]{2}{*}{17} & \multirow{2}{*}{$\begin{array}{c}\text { Gymnema sylvestre } \\
\text { (Retz.) R. Br. exSchultes }\end{array}$} & \multirow[b]{2}{*}{ Asclepiadaceae } & & & Leaves, & Diabetes, \\
\hline & & & Gudmar & Gudmarmarrha & Whole plant, & $\begin{array}{l}\text { Asthma, Snake } \\
\text { bite, }\end{array}$ \\
\hline 18 & Tridax procumbens L. & Asteraceae & Kambarmodi & Kambarmodiarvi & $\begin{array}{l}\text { Leaves, Entire } \\
\text { plant }\end{array}$ & $\begin{array}{l}\text { Cracking foot } \\
\text { wound, Stomach } \\
\text { problems }\end{array}$ \\
\hline 19 & Ageratum conyzoides L. & Asteraceae & Mukhra & Mukhramarrha & Entire plant & stomach disorders \\
\hline 20 & $\begin{array}{c}\text { Oroxylumindicum }(L) \\
\text { Vent }\end{array}$ & Bignoniaceae & Tattu & Tattumarrha & $\begin{array}{l}\text { Fruits and } \\
\text { Flower }\end{array}$ & $\begin{array}{l}\text { Digestive problem, } \\
\text { Masculine }\end{array}$ \\
\hline 21 & $\begin{array}{l}\text { Brassica juncea (L.) } \\
\text { Czern. \& Coss. }\end{array}$ & Brassicaceae & Mohari & Moharimarrha & Leaves, Seeds & $\begin{array}{l}\text { boil. Night } \\
\text { blandness. Skin } \\
\text { diseases. }\end{array}$ \\
\hline & & & & & Fruits, & Cancer, Delivery, \\
\hline 22 & Opuntiaelatior Mill. & Cactaceae & Nagphani & Nagphanimarrha & Bark, Root. & $\begin{array}{l}\text { cough , Skin } \\
\text { disease }\end{array}$ \\
\hline & & & & & Leaf, & Piles, Asthma, \\
\hline 23 & Cassia fistula L. & Caesalpiniacea & $\begin{array}{c}\text { Bahava(Amalta } \\
\text { s) }\end{array}$ & Bahavamarrha & Seeds, & Cough, Diuretic, \\
\hline & & & & & Bark & Cancer, Ghatsarpa \\
\hline
\end{tabular}




\begin{tabular}{|c|c|c|c|c|c|c|}
\hline 24 & Spinacia oleracea L. & Chenopodiaceae & Palak & Palakmarrha & Leaves, & $\begin{array}{l}\text { Blood purifier, } \\
\text { Tonic }\end{array}$ \\
\hline \multirow[t]{2}{*}{25} & \multirow[t]{2}{*}{$\begin{array}{l}\text { Terminalia bellirica } \\
\text { (Gaertn.) Roxb }\end{array}$} & \multirow[t]{2}{*}{ Combretaceae } & \multirow[t]{2}{*}{ Behada } & \multirow[t]{2}{*}{ Behadamarrha } & Fruits, Bark, & $\begin{array}{l}\text { Skin complaints, } \\
\text { Cough, Asthma } \\
\text { and Gastro- } \\
\text { intestinal } \\
\text { problems. }\end{array}$ \\
\hline & & & & & Unripe fruit & $\begin{array}{l}\text { Piles, Cough, } \\
\text { Diuretic, }\end{array}$ \\
\hline \multirow[t]{2}{*}{26} & \multirow[t]{2}{*}{$\begin{array}{c}\text { Terminalia chebula } \\
\text { Retz. }\end{array}$} & \multirow[t]{2}{*}{ Combretaceae } & \multirow[t]{2}{*}{ Hirda } & \multirow[t]{2}{*}{ Hirdamarrha } & Fruit, & $\begin{array}{l}\text { Burning } \\
\text { Sensitization, } \\
\text { Cough, Asthma } \\
\text { and Gastro- } \\
\text { intestinal problem, } \\
\text { Cardiac problems. }\end{array}$ \\
\hline & & & & & Bark, & $\begin{array}{l}\text { Worms, Piles, } \\
\text { Acidity, Cough. }\end{array}$ \\
\hline 27 & Kalanchoe pinnata $L$. & Crassulaceae & $\begin{array}{c}\text { patharchatta or } \\
\text { Panfuti }\end{array}$ & Panfutimarrha & Leaves, & $\begin{array}{l}\text { Kidney Stone. } \\
\text { Stomach pain. }\end{array}$ \\
\hline 28 & $\begin{array}{c}\text { Coccinia grandis } L . \\
\text { Voigt }\end{array}$ & Cucurbitaceae & Tondale & Tondalemarrha & Leaves,Stem & $\begin{array}{l}\text { Skin diseases, } \\
\text { Smallpox }\end{array}$ \\
\hline 29 & Cuscuta reflexa Roxb. & cuscutaceae & Adharwel & Adhrwelmarrha & Whole plant & $\begin{array}{l}\text { Diuretic, Worm, } \\
\text { Pain }\end{array}$ \\
\hline 30 & Dioscorea bulbifera L. & Dioscoreaceae & $\begin{array}{c}\text { Dukkarkanda } \\
\text { (Baichandi) }\end{array}$ & $\begin{array}{c}\text { Dukkarkandamarrh } \\
\text { a } \\
\end{array}$ & Roots & $\begin{array}{l}\text { prolonged } \\
\text { illness,Piles }\end{array}$ \\
\hline \multirow[b]{2}{*}{31} & \multirow[b]{2}{*}{$\begin{array}{l}\text { Dioscorea hispida } \\
\text { Dennst. }\end{array}$} & \multirow[b]{2}{*}{ Dioscoreaceae } & \multirow[b]{2}{*}{ Kadukanda } & \multirow[b]{2}{*}{ kadukandamarrha } & Leaves, & Skin Diseases. \\
\hline & & & & & Roots \& Seeds & $\begin{array}{l}\text { Stomach cleaning, } \\
\text { Purification of } \\
\text { blood. }\end{array}$ \\
\hline 32 & $\begin{array}{l}\text { Bridelia retusa (L.) } \\
\text { Sprang. }\end{array}$ & Euphorbiaceae & Kashi & Telhamarrha & Bark Root & Tonic \\
\hline 33 & Jatropha curcas L. & Euphorbiaceae & Chandrajyot & Kadlavimarrha & Leaves & blood purifier \\
\hline \multirow{4}{*}{34} & \multirow{4}{*}{$\begin{array}{l}\text { Ricinus communis L. } \\
\text { Erand. }\end{array}$} & \multirow{4}{*}{ Euphorbiaceae } & \multirow{4}{*}{ Erandi } & \multirow{4}{*}{ Nerdemarrha } & Seeds, & $\begin{array}{l}\text { Piles, Jaundice, } \\
\text { Rheumatism. }\end{array}$ \\
\hline & & & & & Leaves, & $\begin{array}{l}\text { Oil from seed is } \\
\text { purgative and } \\
\text { used in } \\
\text { rheumatism. }\end{array}$ \\
\hline & & & & & Roots. & $\begin{array}{l}\text { Constipation, } \\
\text { Lactation, } \\
\text { Scorpion sting. }\end{array}$ \\
\hline & & & & & Leaves. & $\begin{array}{l}\text { Joint pain, Brest } \\
\text { tumour. }\end{array}$ \\
\hline \multirow[t]{3}{*}{35} & \multirow[t]{3}{*}{ Phyllanthus urinaria L. } & \multirow[t]{3}{*}{ Euphrbiaceae } & \multirow[t]{3}{*}{ BhuiAwla } & \multirow[t]{3}{*}{ Bhuiawlamarrha } & Whole plants, & $\begin{array}{l}\text { Piles, Viral } \\
\text { hepatitis, } \\
\text { Diarrhoea } \\
\text { dysentery. }\end{array}$ \\
\hline & & & & & Fruits, & $\begin{array}{l}\text { Heart diseases, } \\
\text { Wound, }\end{array}$ \\
\hline & & & & & Latex & White discharge. \\
\hline & & & & & Leaves, & Laryngitis, \\
\hline 36 & Abrus precatorius $L$. & Fabaceae & Gunja & Gunjamarrha & Seeds, & Swelling Pain, \\
\hline & & & & & Roots. & cough. Tonic. \\
\hline 37 & Cajanus cajan (L) & Fabaceae & Toor & Toormarrha & Root & Fever \\
\hline 38 & $\begin{array}{l}\text { Vegna trilobus (L.) } \\
\text { verde. }\end{array}$ & Fabaceae & Ranmung & Ranmungmarrrha & Leaves & Eye diseases \\
\hline & & & & & Seeds, & Dysentery, \\
\hline 39 & $\begin{array}{l}\text { Hepper } \\
\text { Hepro }\end{array}$ & Fabaceae & Mung & Mungmarrha & Seed, & Rheumatism, \\
\hline & & & & & Seed, & Beriberi \\
\hline 40 & $\begin{array}{c}\text { Flacourtia indica } \\
\text { (Burm. f) Merr }\end{array}$ & Flaourtiaceae & Kakai & Kakaimarrha & Bark, Gum & Skin infections \\
\hline 41 & $\begin{array}{l}\text { Curculigo orchiodes } \\
\text { Gaertn. }\end{array}$ & Hypoxitaceae & Kali-musli & kali-muslimarrha & Root & $\begin{array}{l}\text { Aphrodisiac } \\
\text { Hepatoprotective }\end{array}$ \\
\hline & & & & & Leaves, & Asthma, piles, \\
\hline & & & & & Roots., & skin disease. \\
\hline 42 & Vitex nequndo $L$ & Lamiaceae & Nilgudi & Vandamarrha & Root, Leaves, & $\begin{array}{l}\text { Rheumatic } \\
\text { problem. }\end{array}$ \\
\hline 42 & viex reguriar $\mathrm{L}$. & Lamillede & ivingum & valluarlatila & Leaves, & Dysentery, \\
\hline & & & & & Leaves and Bark & $\begin{array}{l}\text { Fever, } \\
\text { Rheumatism, } \\
\text { Scorpion sting. }\end{array}$ \\
\hline & & & & & Plant, & Digestive, \\
\hline 43 & Mentha spicata L. & Lamiaceae & Pudina & Pudinamarrha & $\begin{array}{l}\text { leaves and } \\
\text { flowers }\end{array}$ & Vomiting, Cramps, \\
\hline & & & & & Leaves. & Appendix. \\
\hline 44 & Ocimum sanctum. (L.) & Lamiaceae & Tulsi & Tulsimarrha & $\begin{array}{l}\text { Inflorescence } \\
\text { (flowers) }\end{array}$ & $\begin{array}{l}\text { Weakness, } \\
\text { problem in semen } \\
\text { (thickness) }\end{array}$ \\
\hline 45 & $\begin{array}{c}\text { ButeamonospermaLam } \\
\text { k. Taub }\end{array}$ & Leguminaceae & Palas & Murrimarrha & Leaves & Kidney troubles \\
\hline 46 & Allium sativum & Liliaceae & Lasun & Lasunmarrha & Lasun fruits. & Rheumatism. \\
\hline
\end{tabular}




\begin{tabular}{|c|c|c|c|c|c|c|}
\hline \multirow{3}{*}{47} & \multirow{3}{*}{ Gloriosa superba L. } & \multirow{3}{*}{ Liliaceae } & \multirow{3}{*}{$\begin{array}{l}\text { Kadlavi } \\
\text { (karkari) }\end{array}$} & \multirow{3}{*}{ Kadlavimarrha } & Root, & Syphilic parts, \\
\hline & & & & & seeds, Root, & $\begin{array}{l}\text { Cancer. Abortion, } \\
\text { Child birth, }\end{array}$ \\
\hline & & & & & $\begin{array}{l}\text { leaves, Root, } \\
\text { flower, }\end{array}$ & $\begin{array}{l}\text { AT the time of } \\
\text { delivery, } \\
\text { Inflamation. }\end{array}$ \\
\hline \multirow{3}{*}{48} & \multirow{3}{*}{$\begin{array}{l}\text { Asparagus racemosus } \\
\text { Willd. Var. javanica }\end{array}$} & \multirow{3}{*}{$\begin{array}{c}\text { Liliaceae/Asparagace } \\
\text { ae }\end{array}$} & \multirow{3}{*}{ Satavari } & \multirow{3}{*}{ Satavarimarrha } & Root,\& leaves, & $\begin{array}{l}\text { Urinary problems, } \\
\text { Weakness, }\end{array}$ \\
\hline & & & & & Roots, leaves, & $\begin{array}{l}\text { Dysentery, Night } \\
\text { blindness, }\end{array}$ \\
\hline & & & & & Root, leaves. & $\begin{array}{l}\text { Nutrition. } \\
\text { Lac of breast milk }\end{array}$ \\
\hline 49 & $\begin{array}{c}\text { Pongamia pinnata }(\mathrm{L} .) \\
\text { Pierre }\end{array}$ & Luguminosae & Karanj & Karanjimarrha & Seed, Bark & Wound, Arthritis \\
\hline \multirow[t]{2}{*}{50} & \multirow[t]{2}{*}{ Punica granatum $L$. } & \multirow[t]{2}{*}{ Lythraceae } & \multirow[t]{2}{*}{ Darim } & \multirow[t]{2}{*}{ Darimmarrha } & Fruit,Seeds, & $\begin{array}{l}\text { Digestive problem, } \\
\text { Jaundice and } \\
\text { diarrhoea. }\end{array}$ \\
\hline & & & & & Seeds. & $\begin{array}{l}\text { Urinary track inf. } \\
\text {,Indigestion. }\end{array}$ \\
\hline 51 & Lawsonia inermis L. & Lythraceae & Mehandi & Mehandimarrha & Leaves, & $\begin{array}{l}\text { Useful in cough } \\
\text { and vomiting. } \\
\text { Headache, } \\
\text { Rheumatism, Hair } \\
\text { growth \& hair die, } \\
\text { Burning feet, } \\
\text { Wound \& ulcer. }\end{array}$ \\
\hline \multirow[b]{2}{*}{52} & \multirow[b]{2}{*}{$\begin{array}{c}\text { Abelmoschus } \\
\text { esculentus (L.)Moench }\end{array}$} & \multirow[b]{2}{*}{ Malvaceae } & \multirow[b]{2}{*}{ Bhendi } & \multirow[b]{2}{*}{ Bhendimarrha } & Root, & Sex power \\
\hline & & & & & Whole plant & $\begin{array}{l}\text { cut wound, } \\
\text { Abdominaldisorde } \\
\text { rs }\end{array}$ \\
\hline 53 & $\begin{array}{c}\text { Malvastrum } \\
\text { coromandelianum (L.) } \\
\text { Garcke }\end{array}$ & Malvaceae & $\begin{array}{l}\text { Ran-chikna } \\
\text { (shendri) }\end{array}$ & Ran-chiknamarrha & Leaves & Diabetes \\
\hline \multirow[b]{2}{*}{54} & \multirow[b]{2}{*}{ Martynia annua L. } & \multirow[b]{2}{*}{ Martyniaceae } & \multirow[b]{2}{*}{ Wagnakhi } & \multirow[b]{2}{*}{ wagnakhimarrha } & Fruit, & Snake bite, \\
\hline & & & & & $\begin{array}{l}\text { Root, Whole } \\
\text { plant. }\end{array}$ & $\begin{array}{l}\text { Anti- } \\
\text { inflammatory, } \\
\text { Menstrual } \\
\text { disorder }\end{array}$ \\
\hline \multirow{5}{*}{55} & \multirow{5}{*}{$\begin{array}{c}\text { Azadira chtaindica } \\
\text { Juss. }\end{array}$} & \multirow{5}{*}{ Meliaceae } & \multirow{5}{*}{ Kaduneem } & \multirow{5}{*}{ Epamarrha } & $\begin{array}{l}\text { Leaves, Whole } \\
\text { plant, } \\
\text { Bark, stem, }\end{array}$ & $\begin{array}{l}\text { Fever,Gonorrhea, } \\
\text { Toothache, } \\
\text { Blood purifire, }\end{array}$ \\
\hline & & & & & $\begin{array}{l}\text { Bark, leaf, } \\
\text { Stem bark, }\end{array}$ & $\begin{array}{l}\text { Tapeworm., } \\
\text { Asthma, cough, } \\
\text { leprosy, Acidity, }\end{array}$ \\
\hline & & & & & $\begin{array}{l}\text { leaves, Leaves, } \\
\text { Leaves, Leaves, } \\
\text { stem, }\end{array}$ & $\begin{array}{l}\text { Tooth Powder, } \\
\text { Anemia, Diabetes, } \\
\text { Malaria, leprosy } \\
\text { \&leucoderma. }\end{array}$ \\
\hline & & & & & $\begin{array}{l}\text { Leaves,stem,Bar } \\
\text { k, stem, }\end{array}$ & $\begin{array}{l}\text { Jaundice \&skin } \\
\text { diseases. }\end{array}$ \\
\hline & & & & & $\begin{array}{l}\text { Leaves, seed, } \\
\text { Root. Bark, }\end{array}$ & $\begin{array}{l}\text { Blood problems } \\
\text { wayu Pitta } \\
\text { andcough } \\
\text { remover. }\end{array}$ \\
\hline & & & & & Leaves, & Fever, Skin \\
\hline 56 & Melia azadirachta L. & Meliaceae & Mahaneem & Mahaneemmarrha & Bark, & disease, leprocy, \\
\hline & & & & & Leaves, Flower & Hysteria, Kille lice \\
\hline 57 & $\begin{array}{l}\text { Soymidaf ebrifuga } \\
\text { (Roxb.) Juss. }\end{array}$ & Meliaceae & $\begin{array}{c}\text { Raktrohan } \\
\text { (chitrak) }\end{array}$ & Raktrohanmarrha & Roots, bark & $\begin{array}{l}\text { Blood pressure } \\
\text { chest pain. }\end{array}$ \\
\hline 58 & $\begin{array}{l}\text { Cocculus hirsutus } \\
\text { (L.) Theob. }\end{array}$ & Menispermaceae & Wasanvel & Wasanvelmarrha & Root.Leaves. & $\begin{array}{l}\text { Pediatrics bowl } \\
\text { disorder. } \\
\text { Dhaturog }\end{array}$ \\
\hline & & & & & Seeds, & digestive troubles, \\
\hline & & & & & $\begin{array}{l}\text { Stem Bark paste. } \\
\text { Bark, }\end{array}$ & $\begin{array}{l}\text { Toothache, } \\
\text { Throatinfection, }\end{array}$ \\
\hline 59 & $\begin{array}{l}\text { Acacia niloticaL. Willd. } \\
\text { Ex Del. Ssp. Indica } \\
\text { (Bth) Brenan }\end{array}$ & Mimosaceae & Babul & Tummamarrha & Leaves, Root, & $\begin{array}{l}\text { Acidity, Fever, } \\
\text { Diarrhoea, } \\
\text { disentry }\end{array}$ \\
\hline & & & & & $\begin{array}{l}\text { Fruit, Gum, } \\
\text { Bark }\end{array}$ & $\begin{array}{l}\text { anddiabetes. } \\
\text { eyesconjucticity to } \\
\text { stop lacrimination }\end{array}$ \\
\hline & & & & & Leaves, whole & $\begin{array}{l}\text { Rheumatism.Piles. } \\
\text { Dysentery, }\end{array}$ \\
\hline 60 & Mimosa Pudica L.. & Mimosacease & Lajalu/Lajvanti & Lajalumarrha & plant, Root, & $\begin{array}{l}\text { Jaundice, U.T.I, } \\
\text { Cough. }\end{array}$ \\
\hline 61 & Acacia farnesiana (L.) & Mimosoideae & Khair & Khairmarrha & Seed, Bark, & Kidney troubles, \\
\hline
\end{tabular}




\begin{tabular}{|c|c|c|c|c|c|c|}
\hline & Willd. & & & & $\begin{array}{l}\text { Bark, Root, } \\
\text { Bark, Bark }\end{array}$ & $\begin{array}{l}\text { Indigestion, } \\
\text { Snakebite, } \\
\text { Wound, Mouth } \\
\text { infection, } \\
\text { Toothache }\end{array}$ \\
\hline 62 & $\begin{array}{l}\text { Glinusop positifolius } \\
\text { (L.) A.DC. }\end{array}$ & Molluginaceae & kadubhaji & KaytaBhaji & Leaves & Vitamin deficiancy \\
\hline 63 & Mimosa Pudica L.. & Momosaceae & Lajalu & Lajulimarrha & Leaves & Skin \\
\hline 64 & Streblus asper Lour. & Moraceae & Kharasani & Kharasanimarrha & $\begin{array}{l}\text { Small branches, } \\
\text { root, bark. }\end{array}$ & $\begin{array}{l}\text { Burning Skin, } \\
\text { Toothache, } \\
\text { dysentery }\end{array}$ \\
\hline \multirow{4}{*}{65} & \multirow{4}{*}{$\begin{array}{c}\text { Ficus benghalensis } \\
\text { Linn. L. }\end{array}$} & \multirow{4}{*}{ Moraceae } & \multirow{4}{*}{ Wad } & \multirow{4}{*}{ Wadmarrha } & Bark, & Bowel Complaints. \\
\hline & & & & & leaves, & $\begin{array}{l}\text { Rheumatism, } \\
\text { Diabetes, } \\
\text { Gonorrhea, }\end{array}$ \\
\hline & & & & & $\begin{array}{c}\text { Bark, Root, } \\
\text { Buds, }\end{array}$ & $\begin{array}{l}\text { Dysentery, } \\
\text { Haemoptysis. } \\
\text { Diabetes, Stomach } \\
\text { pain, Inflamation. } \\
\text { U.T.I., }\end{array}$ \\
\hline & & & & & Bark, & \begin{tabular}{|l|} 
Gonorrhea, \\
Fistula, Skin \\
diseases. \\
\end{tabular} \\
\hline 66 & Ficus religiosa $\mathrm{L}$. & Moraceae & Pimpal & Pimpalmarrha & Bark & $\begin{array}{l}\text { Dysentery and } \\
\text { Diarrhoea, } \\
\text { Rheumatism } \\
\text { Germicidal } \\
\text { properties. }\end{array}$ \\
\hline \multirow{4}{*}{67} & \multirow{4}{*}{ Moringa oleifera Lam. } & \multirow{4}{*}{ Moringaceae } & \multirow{4}{*}{ Shevga } & \multirow{4}{*}{ Shevgamarrha } & Fruits, seed, & $\begin{array}{l}\text { Rheumatism, } \\
\text { various disease. }\end{array}$ \\
\hline & & & & & flowers, & Sore throat, \\
\hline & & & & & Root, & Headache, \\
\hline & & & & & Seed, & Diuretic. \\
\hline \multirow{3}{*}{68} & \multirow{3}{*}{$\begin{array}{c}\text { Eucalyptus globulus L } \\
\text { Herit. }\end{array}$} & \multirow{3}{*}{ Myrtaceae } & \multirow{3}{*}{ Nilgiri } & \multirow{3}{*}{ Nilgirimarrha } & Leaves, Leaves, & $\begin{array}{l}\text { Stimulus for } \\
\text { Sexual power, }\end{array}$ \\
\hline & & & & & Stem, & Headache, \\
\hline & & & & & Leaves, & $\begin{array}{l}\text { Cold, cough, } \\
\text { Headache, }\end{array}$ \\
\hline \multirow{3}{*}{69} & \multirow{3}{*}{$\begin{array}{l}\text { Syzygiumcumini (L.) } \\
\text { Skeels }\end{array}$} & \multirow{3}{*}{ Myrtaceae } & \multirow{3}{*}{ Jambhul } & \multirow{3}{*}{ Nedimarrha } & Leaves, & Diabetes. \\
\hline & & & & & fruits. & Diarrhoea and \\
\hline & & & & & Seed, Fruit, & dysentery, Tonic \\
\hline 70 & Nelumbo nucifera L. & Nelumbonaceae & Kamal & kamalmarrha & Flower, & $\begin{array}{l}\text { Diarrhoea, Tonic, } \\
\text { High Blood } \\
\text { Pressure. }\end{array}$ \\
\hline 71 & Boerhavia diffusa $L$. & Nyctaginaceae & $\begin{array}{l}\text { Punarnava } \\
\text { (khaparKutti) }\end{array}$ & Punarnavamarrha & Whole plant & $\begin{array}{l}\text { worms, } \\
\text { cough.Malaria, } \\
\text { Asthma, } \\
\text { Jauindice, } \\
\text { Leucorrhoea }\end{array}$ \\
\hline \multirow[b]{2}{*}{72} & \multirow{2}{*}{$\begin{array}{l}\text { Bougainvillea } \\
\text { spectabills Willd. }\end{array}$} & \multirow[b]{2}{*}{ Nyctaginaceae } & \multirow[b]{2}{*}{ Boganwel } & \multirow[b]{2}{*}{ Boganwelmarrha } & Leaves,Leaves, & Diarrhoea, Acidity, \\
\hline & & & & & Flower & $\begin{array}{l}\text { Lucorrhoea,Blood } \\
\text { Pressure. }\end{array}$ \\
\hline 73 & $\begin{array}{c}\text { Jasminum auriculatum } \\
\text { Vahl }\end{array}$ & Oleaceae & Kunda & Kundamarrha & Flower, & Hair growth \\
\hline 74 & Jasminum officinale $L$. & Oleaceae & Chameli & Chamelimarrha & Flower, Leaves, & $\begin{array}{l}\text { Pain, Menstrual } \\
\text { disorder, cough,. }\end{array}$ \\
\hline 75 & $\begin{array}{c}\text { Vanda te stacea (Lindl.) } \\
\text { Reichb.f. }\end{array}$ & Orchidaceae & Vanda(Rasana) & Vandamarrha & Leaves, root & $\begin{array}{l}\text { Appetizer, cough, } \\
\text { Chest pain, } \\
\text { asthma. } \\
\text { Rheumatism and } \\
\text { problem of } \\
\text { nervous system. }\end{array}$ \\
\hline 76 & $\begin{array}{c}\text { Atylosia scarabaeoides } \\
\text { (L.) Benth. }\end{array}$ & Papilionatae & Rantur & Ranturmarrha & Pods & $\begin{array}{l}\text { Abdominal } \\
\text { problems, Wounds }\end{array}$ \\
\hline 77 & $\begin{array}{c}\text { Butea superb Roxb. Ex } \\
\text { Willd. }\end{array}$ & Papilionatae & Palasvel & Murriveli & Gum & $\begin{array}{l}\text { Prolonged } \\
\text { weakness }\end{array}$ \\
\hline 78 & Sesamum indicum $L$. & Pedaliaceae & Til & Tilmarrha & Seed,Leaves, & $\begin{array}{l}\text { cough, Kidney } \\
\text { stone }\end{array}$ \\
\hline \multirow{4}{*}{79} & \multirow{4}{*}{ Ficus racemose L. } & \multirow{4}{*}{ Moraceae } & \multirow{4}{*}{ Umber } & & Roots, & Kidney \\
\hline & & & & Umbermarrha & Fruits., & Stomach troubles, \\
\hline & & & & UMDermarrna & Fruit, & Inflamation, \\
\hline & & & & & Fruit. & U.T.I., \\
\hline 80 & $\begin{array}{l}\text { Cynodon dactylon } \\
\text { (L.)Pers. }\end{array}$ & Poaceae & Durva & Durvamarrha & $\begin{array}{l}\text { Root, Whole } \\
\text { Plant }\end{array}$ & $\begin{array}{l}\text { Gonorhya, } \\
\text { Burning } \\
\text { sensation, } \\
\text { Diarrhoea, Brain } \\
\text { Tonic, }\end{array}$ \\
\hline
\end{tabular}




\begin{tabular}{|c|c|c|c|c|c|c|}
\hline 81 & Portulaca oleracea L. & Portulacaceae & Ghol & Gholmarrha & Whole plant & $\begin{array}{l}\text { Tooth bleeding, } \\
\text { burning sensation. } \\
\text { Fever,Acidity. }\end{array}$ \\
\hline 82 & $\begin{array}{c}\text { Citrus limon (L). Burm. } \\
\text { f. }\end{array}$ & Rutaceae & Limbu & Limbumarrha & Fruit & $\begin{array}{l}\text { Burning in } \\
\text { urination } \& \\
\text { constipation }\end{array}$ \\
\hline 83 & $\begin{array}{c}\text { Ceriscoide sturgida } \\
\text { (Roxb) Tirveng }\end{array}$ & Rhubiaceae & Pandhrafendra & Madanghanta & Fruits & Digestive problem \\
\hline 84 & $\begin{array}{l}\text { Adina cordifolia (Roxb.) } \\
\text { Hook f. ex Brandis. }\end{array}$ & Rubiaceae & Haldu & $\begin{array}{l}\text { Haldimarrha, } \\
\text { Kamakamarrah }\end{array}$ & Leaf & Fever \\
\hline 85 & $\begin{array}{c}\text { Anthocephalus } \\
\text { cadamba (Roxb.) Miq. }\end{array}$ & Rubiaceae & Kadamb & Kadambmarrha & Leaves, Bark, & $\begin{array}{l}\text { Appetizer, } \\
\text { Snakebite, Uterus } \\
\text { contraction }\end{array}$ \\
\hline 86 & $\begin{array}{l}\text { Feronia limonia } L . \\
\text { swingle }\end{array}$ & Ruraceae & kawath & kawatmarrha & Fruits and leaves & Shwetpardar \\
\hline 87 & $\begin{array}{l}\text { Murraya koenigii L. } \\
\text { Spreng }\end{array}$ & Rutaceae & $\begin{array}{l}\text { Kaddhipatta } \\
\text { (Godlimb) }\end{array}$ & Kaljamarrha & Leaves, Roots, & $\begin{array}{l}\text { Digestion, Skin } \\
\text { diseases, Stomach } \\
\text { diseases, } \\
\text { Jaundice, }\end{array}$ \\
\hline 88 & $\begin{array}{l}\text { Cardiospermum } \\
\text { halicacabum } \mathrm{L} .\end{array}$ & Sapindaceae & Kapalphodi & Kapalphodimarrha & $\begin{array}{l}\text { Leaves and } \\
\text { roots. }\end{array}$ & $\begin{array}{l}\text { Ear ache, wound } \\
\text { and in arthritis. } \\
\text { Dysentery }\end{array}$ \\
\hline 89 & $\begin{array}{c}\text { SapindustrifoliatusHier } \\
\mathrm{n} .\end{array}$ & Sapindaceae & Kusum & Kusummarrha & Seed & $\begin{array}{l}\text { Skin disease, } \\
\text { Ulcers. }\end{array}$ \\
\hline 90 & $\begin{array}{c}\text { Bacopa monnieri (L.) } \\
\text { Penn. }\end{array}$ & Scrophulariaceae & Bhrami & Bhramimarrha & Whole palnt & $\begin{array}{l}\text { Epilepsy Fever, } \\
\text { Brain Tonic, } \\
\text { Rhumatism, } \\
\text { Diarrhea, } \\
\text { Abdomenal } \\
\text { Diseases }\end{array}$ \\
\hline 91 & Scoparia dulcis L. & Scrophulariaceae & $\begin{array}{l}\text { Ranmethi/ } \\
\text { ghadtulsh }\end{array}$ & Ranmethimarrha & Complete plant & diabetes. \\
\hline 92 & Ailanthus excels Roxb. & Simarubaceae & Maharukh & Maharukhmarrha & leaves, Bark. & $\begin{array}{l}\text { leucoderma, } \\
\text { itching, cholera } \\
\text { and asthma, } \\
\text { killing worm }\end{array}$ \\
\hline 93 & Datura inoxia L. & Solanaceae & $\begin{array}{c}\text { PandharaDhotr } \\
\text { a }\end{array}$ & PandharaDhotra & Seed & pimples \\
\hline 94 & $\begin{array}{l}\text { Lycopersicon } \\
\text { esculentum Mill. }\end{array}$ & Solanaceae & Tamater & Tamatermarrha & Leaves, & $\begin{array}{l}\text { Skin diseases, } \\
\text { Bend (Galandd) }\end{array}$ \\
\hline \multirow{3}{*}{95} & \multirow{3}{*}{ Helictere sisora. $L$. } & \multirow{3}{*}{ Sterculiaceae } & \multirow{3}{*}{ Muradsheng } & \multirow{3}{*}{ Muradshengmarrha } & Root, & Diabetes, \\
\hline & & & & & Bark, & Dysentery, \\
\hline & & & & & Fruit, & Stomach ache, \\
\hline 96 & Sterculia urens Roxb. & Sterculiaceae & $\begin{array}{c}\text { Karu } \\
\text { (Kahandol) }\end{array}$ & karumarrha & Gum & $\begin{array}{l}\text { Leprosy, Pregnant } \\
\text { ladies diet. }\end{array}$ \\
\hline 97 & $\begin{array}{c}\text { Cissus quadrangularis } \\
\text { L. }\end{array}$ & Vitaceae & $\begin{array}{l}\text { Kandvel } \\
\text { (hadjod) }\end{array}$ & Kandvelimarrha & Stem, Root & $\begin{array}{l}\text { Diuretic, Fracture } \\
\text { bone, Wound \& } \\
\text { cut }\end{array}$ \\
\hline 98 & Curcuma longa L. & Zingiberaceae & $\begin{array}{l}\text { Halkand/Halad } \\
\text { (Turmeric) }\end{array}$ & Halkandmarrha & Rhizome,Root & $\begin{array}{l}\text { Rheumatism, } \\
\text { Arthritis. } \\
\text { Cold,Wound, } \\
\text { Fever }\end{array}$ \\
\hline \multirow{6}{*}{99} & \multirow{6}{*}{$\begin{array}{c}\text { Zingiber officinale } \\
\text { Boehm. }\end{array}$} & \multirow{4}{*}{ Zinziberaceae } & \multirow{4}{*}{$\begin{array}{l}\text { Adrak/Ala/ } \\
\text { Sunth (dried } \\
\text { rhizome) }\end{array}$} & \multirow{4}{*}{ Adrak } & Leaves, & Tooth decaying, \\
\hline & & & & & Flower, & Fever, \\
\hline & & & & & Zinger & Asthma, Cough, \\
\hline & & & & & Rhizome, & Indigestion, \\
\hline & & & & & Stem, & Inflammation, \\
\hline & & & & & Root. & Vomiting. \\
\hline $\begin{array}{c}10 \\
0\end{array}$ & Tribulusterrestris $L$. & Zygophyllaceae & Gokharu & Gokharumarrha & Fruit, & $\begin{array}{l}\text { Sex power, Tooth } \\
\text { ache, U.T.I }\end{array}$ \\
\hline
\end{tabular}

\section{Results and Discussion:}

The present study was aimed to investigate the plants used by the local and tribal people for their medicinal values. The given list of the some important medicinal plants found in the Kurkheda and Korchi forest region along with their Local names(s), family, distribution, parts used and ethnomedicinal uses. The present study records 100 species of ethno-medicinal plant representing 60 families used for medicinal purposes by local and tribal people were documented. Knowledge regarding the occurrence and availability of selected species was obtained from the local people through participation, either by interview, or workshop Semi-structured interview. A brief information including botanical name, family, local name, part used and their medicinal value is presented here. The tribal villagers are using these plant to cure many diseases, like Urinogenital disorder, Menstrual disorder, Hypertension, Cough, Diarrhoea, Dysentery, Wound healing, Diabetes, Jaundice, Sunstroke, Fever, Skin diseases Abortificients and as blood purifier etc. These people prepare the medicine as decoction, oral treatment, ointment etc. The extract and the paste are the two main source of methods for treatment of diseases. However, the use of a 
particular plant part depends on the plant habit and user's needs. The use of specific plant parts for the treatment suggests that these parts have strongest medicinal properties but it needs conformation of botanical analysis and pharmaceutical screening to cross-check the local information. Our findings of the frequent use of green leaves and fruit in the preparation of remedies corroborate the result. The Liquid such as water juices, Jaggery, tea, honey, mustered oil, ghee and milk are mixed with plants or plant part during the preparation of the remedies. The prepared remedies are mostly administered orally $(77 \%)$, less frequently dermally $(10 \%)$ or both orally and dermally $(12 \%)$. Only $1 \%$ is administered through ears or eyes.

\section{Conclusion:}

The forest range in Gadchiroli district is very rich in commercially and pharmaceutically important ethnomedicinal plant species. The traditional healers have old knowledge regarding the uses of medicinal plants and they practice traditional way of curing a wide spectrum of diseases. Few species were found to be under threat probably due to over collection. Especially perennial herbs with rhizomes are of conservation concern. The local inhabitants depend on plant, for the treatment of diseases but not all are familiar with the proper collection methods, parts to be used, preservation and storage. In contrast, local traditional healers are familiar with proper collection and use of medicinal plant and they should be involved in efforts, of conservation and sustainable use of ethnomedicinal plant resources.

\section{Acknowledgement:}

Authors are thankful to the forest officials of Kurkheda and Korchi forest range and the local people of the village for the information.

\section{References:}

1. Ahir. R, Pokale. S, W agh. S.

Studies on biodiversity of certain medicinal plants ofAhmadnagar region, M.S., India. Indian Stream Research Journal., 2011, 1(6), 1-4.

\section{Bakare. S.}

Ethnomedicinal Plants Diversity AroundNawargaon Village Of Chandrapur District, Maharashtra, India, Weakly Science Research Journal., 2014,Vol 1(24).

3. Bako. S, Bakfur. M. J, John. I, Bala. E I.

Ethnomedicinal and phytochemical profile of some savanna plant species in Nigeria. Int. J. Bot, 2005, 1:147-150.

4. Bhat. J. A, Kumar. M, Bussmann. R.W.

Ecological status and traditional knowledge of medicinal plants in Kedarnath Wildlife Sanctuary of
Garhwal Himalaya India.J Ethnobiol Ethnomed, 2013, 9:1.

5. Borkar. Lekhram, Borkar. Laxmikant,

Mate. D.M. Ethno botanical importance of some plants of Euphorbiaceae in Gadarwara Tehsil (M.P.). J. Sci. Infor. Special.,2013 Vol. (6): 24-27.

\section{Burlakoti. C,Kunwar. R.M.}

Folk herbal

medicine of Mahakali watershed area, Nepal. In: Medicinal plant in Nepal: An anthology of contemporary research. (eds.) P.K. Jha, S.B. Karmacharya, M.K. Chhetri, C.B. Thapa and B.B. Shrestha. Ecological Society, Kathmandu., 2008, Nepal, pp. 187-193.

7. Chavhan.P. R.

Diversity of Medicinal

Plants in and Around Etapalli Forest Range in Gadchiroli District. The Journal of Agriculture and Natural Resources Sciences.,2014, 1(2):114-117

8. Dhore. M.M, Dabhadkar.D.D, Zade.

V.S, Dhore. M, Documentation of fertility regulatory ethnomedicinal plants used by tribals of Yavatmal district, Maharashtra, India. Int. J. Sci. \& Res. Pub., 2012, 2(3): 1-6.

\section{Gond, Gopal.}

Ethnobotanical study ofplants by the traditional users of Ballarpur and Gondpipari area of Chandrapur district with reference to their conservation. J. Sci. Infor., 2013, 6: 186-188.

\section{Pocchi. V.}

Ethno-veterinary medicinal plants and its conservation status in the Buldhana District. J. Sci. Infor., 2013, 6:44-47.

11. Harney.N.V.

Ethnomedicinal Plants Diversity of BhadrawatiTahsil of Chandrapur District, Maharashtra, India. IJSRP., 2013, Vol.3 (8): 1-6.Iqbal et al.,(2010).

\section{Kala. C.P.}

Ethnomedicinal botany of the Apatani in the eastern Himalayan region of India. J EthnobiolEthnomed., 2005, 1:11-18.

14. Kumari. P, Joshi. G. C, L. M. Tewari.

Diversity and status of ethno-medicinal plants of Almora district in Uttarakhand, India. International Journal of Biodiversity and Conservation. 2011, Vol. 3(7), pp. 298-326.

\section{Kunwar. R. M, Dawadee. N.P.}

Ethnobotanical notes on flora of Khaptaed National Park, Far-Western Nepal. Him. J. Sci.,2003, 1: 25-30

\section{M uthu. C, Ayyanar. M, Raja. N,}

Ignacimuthu. S. Medicinal plants used by traditional healers in Kancheepuram District of Tamil Nadu, India. J Ethnobiol Ethnomed., 2006, 2:43-53

\section{Shrirame. A.M, Hiwale. S.R.}

Ethnomedicinal Survey for Important Plants of Kalmeshwartaluks, District Nagpur. ISRJ., 2013, pp. 29-31.

\section{Singh. R, Singh. S.}

Documentation of Medicinal Plants from SV Govt. Degree \& PG College Campus Palem, Mahabubnagar Dist. Telangana, India. American Journal of Ethnomedicine, 2014, Vol. 1, No. 6

19. Ugemuge. N.R. 
Flora of Nagpur district, Shree Prakashan Nagpur, 1985.

20. Wadekar. M.B, Tondare.M .J,Rangari.

N.U. Ethnomedicinal plant wealth used for the treatment of the Jaundice by the tribal communities of Chandrapur, District (MS). J. Sci. Infor., 2013, 6:159164.

21. W atile. V.J.

Diversity of medicinal plants use by tribes in Kelapurtahsil of Yavatmal district, - A case study.ISRJ., 2013, pp. 94-96.
22. WHO: Traditional medicine, growing needs and potential. WHO Policy Perspectives on Medicines 2002, 2:1-6. 23.

\section{3] Zingare. A. K, Borkar. K. M, Jagiya.}

A.A. Ethnoveterinary Use of Medicinal Plants from SakoliTaluka of Bhandara District, M. S. ISRJ., 2013, pp. 22-24.

24. Zingare. A.K.

Ethnomedicinal plant diversity of Sakolitaluka of Bhandara district (M.S.). J. Sci. Infor., 2012, 3:5869. 